\title{
Minimising Collisions in RFID Data Streams using Probabilistic Cluster-Based Technique
}

\author{
Prapassara Pupunwiwat • Bela Stantic
}

Received: 28 February 2011 / Accepted: 30 July 2012

\begin{abstract}
Radio Frequency Identification (RFID) uses wireless radio frequency technology to automatically identify tagged objects. Despite the extensive development of the RFID technology in many areas, tags collisions still remain a major problem. This issue is known as the collision problem and can be solved by using anti-collision techniques. Current probabilistic anticollision approaches suffer from tag starvation due to the inaccurate Backlog estimation and have a low performance in some cases. In this research, we propose a Probabilistic Cluster-Based Technique (PCT) to maximise the performance efficiency during the tag identification process. The PCT approach creates new tag grouping strategies using particular equations, according to the optimal efficiency obtained for a specific number of tags. Through extensive experimentation, we have demonstrated that the proposed concept performs better than the other current state-of-the-art approaches.
\end{abstract}

Keywords Radio Frequency Identification (RFID) . Anti-Collision · Probabilistic

\section{Introduction}

RFID technology is an automated wireless technology that uses radio frequency waves to track items. It has the potential to improve the efficiency of business processes by providing automatic identification and data capture. In modern day, there are various applications where RFID technology is integrated such as warehouse

Prapassara Pupunwiwat, Bela Stantic

Griffith University, Gold Coast Campus

Queensland 4222, Australia

Tel.: +617-555-28761

Fax: +617-555-28066

E-mail: Paz.Pupunwiwat@gmail.com,B.Stantic@griffith.edu.au and supply chain monitoring. In those applications where numerous RFID tags are presented in the interrogation zone simultaneously, the RFID reader is required to have an ability to read data from individual tags. If more than one tag tries to communicate to the reader at the same time, a collision occurs and the tag will need to be re-transmitted. A technical approach that handles tag collision without any interference is called an anti-collision scheme.

Several methodologies have been proposed to minimise collision issues in RFID system. The two types of tag anti-collision methods widely used are the Deterministic and the Probabilistic approaches. Deterministic methods perform the tag identification by transmitting a query with specific prefixes, and the tags respond with their ID. This procedure leads to lengthy queries issued by the reader and causes identification delay. On the other hand, probabilistic methods decrease the probability of collision by scheduling the responses of tags at random time. This process leads to tag starvation problems as not all tags can be identified after the long waiting period of time.

In this study, we propose a new method called a Probabilistic Cluster-Based Technique (PCT), to minimise the total number of slots and frames queried during the tag identification process and to maximise the system efficiency. In order to show the significance of our proposed methods, we conducted an experimental evaluation and compared our methods to the existing techniques. The results from our experimentation studies indicate that the proposed PCT256 method maintained its system efficiency above other existing methods and has the highest achieving performance.

The remainder of this paper is organised as follows: in section 2, we provide general background information related to tag collisions and different anti-collision 
schemes. Section 3 contains discussion of the different probabilistic anti-collision methods along with Backlog estimation techniques and their limitations. We present our new methodology, the Probabilistic Cluster-Based Technique in section 4 . In section 5 , we present our experimental evaluation, results and analysis; and finally, we conclude the paper in section 6 .

\section{RFID Background}

RFID technology is an automatic identification technology that identifies electronic tags attached to items without the need for a direct line of sight between the reader and the tag. There are several methods of identification but the most common is to store data containing the tag's identifier known as EPC (Electronic Product Code).

RFID system may only consist of a tag and a reader but a complete RFID structure involves many other components and software such as computer, network, Internet, middleware, and user applications. RFID reader retrieves information from tags and sends that information back to host computer via middleware. RFID data streams that are captured by readers can be accumulated very fast, resulting in data collisions. While there are approaches previously proposed in literature, based on identifying and rectifying the missing observational data after it has been stored within the database [4], it is crucial that the RFID system must employ anticollision protocols in readers in order to enhance the integrity of captured data.

\subsection{Tag and Reader Collisions}

Simultaneous transmissions in RFID systems lead to collisions as the readers and tags typically operate on the same channel. Three types of collisions are possible: Reader-Tag collision, Tag-Tag collision, and ReaderReader collision [10].

- Reader-to-Tag: Interference occurs when one tag is simultaneously located in the interrogation zone of two or more readers, where more than one reader attempts to communicate with that tag at the same moment.

- Tag-to-Tag: Tag collision in RFID systems happens when multiple tags are energised by the RFID reader simultaneously, and reflect their respective signals back to the reader at the same time. This problem is often seen whenever a large volume of tags must be read together in the same reader zone because the reader is unable to differentiate these signals.
- Reader-to-Reader: Interference occurs when a reader transmits a signal that interferes with the operation of another reader and prevents the second reader from communicating with tags in its reader zone. Reader-to-Reader collision can be avoided by determining the appropriate reader's deployment that prevents direct signal interference between two or more readers.

\subsection{Tag Anti-Collision Approaches}

Tag collision problem is more complex than those within reader collision categories. The various types of tag anti-collision approaches for tag collision can be reduced to two basic types: deterministic approaches and probabilistic approaches.

\subsubsection{Deterministic Approaches}

The deterministic approach starts by asking for the first binary bit of the tag until it matches the tags; then it continues to ask for additional characters until all tags within the region are found. This approach is slow and introduces an identification delay but leads to fewer collisions, and has 100 percent successful identification rate [16], [21], [17], [19].

\subsubsection{Probabilistic Approaches}

In a probabilistic approach, tags respond at randomly generated times. If a collision occurs, colliding tags will have to identify themselves again after waiting a random period of time. This technique is faster than deterministic based approach but suffers from tag starvation problem where not all tags can be identified due to the random nature of chosen time.

When we mentioned the probabilistic anti-collision approach in RFID, we usually refer to the ALOHAbased approach, which is the most widely used type of anti-collision. Slotted ALOHA [20], which initiates discrete time-slots for tags to be identified by reader at the specific time, was first employed as an anti-collision method in an early days of RFID technology. The principle of Slotted ALOHA techniques is based on the Pure ALOHA introduced in early 1970s [1], where each tag is identified randomly. To improve the performance and throughput rate, different anti-collision schemes were suggested in the past literature. The three most accepted techniques, Basic Framed-Slotted ALOHA, Dynamic Framed-Slotted ALOHA, and Enhanced Dynamic Framed-Slotted ALOHA, are described in the next section. 


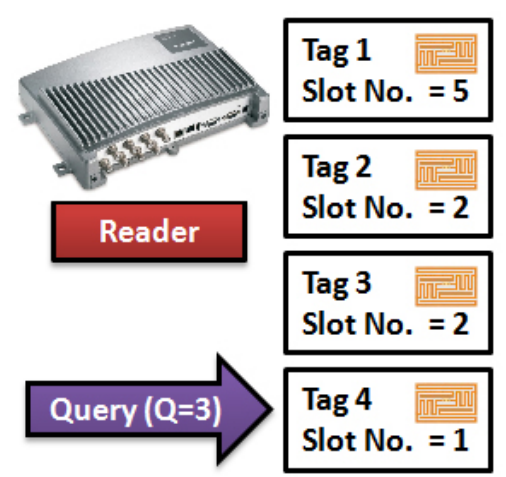

a)

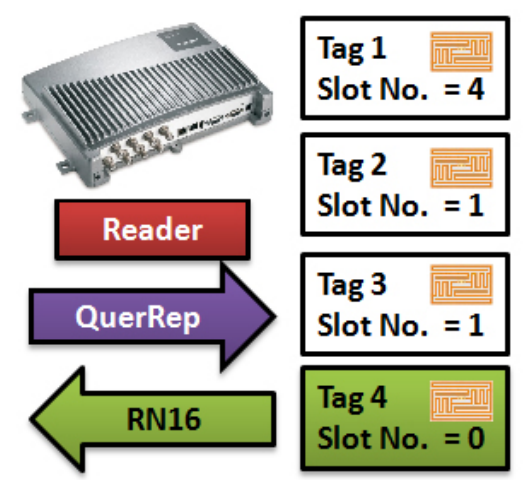

b)

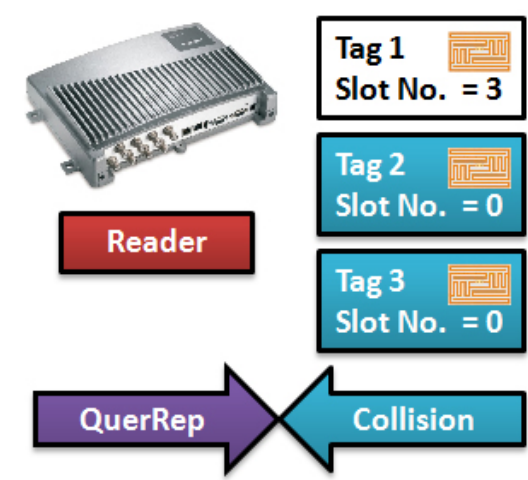

c)

Fig. 1 a) Empty Slot; b) Successful Slot; and c) Collision Slot in EPC Class 1 Gen2 Protocol

\section{ALOHA-Based Anti-collision Approaches}

This section explains the fundamental of Basic FramedSlotted ALOHA, Dynamic Framed-Slotted ALOHA, Enhanced Dynamic Framed-Slotted ALOHA, and Backlog estimation techniques.

\subsection{Basic Framed-Slotted ALOHA Algorithm}

The Basic Framed-Slotted ALOHA (BFSA) is the most basic of all the algorithms that use a fixed frame-size throughout the identification round. The reader offers information to the tags including the frame-size specification and the random number selected by each slot within the frame. Each tag selects a slot using the random number and then sends its ID back to the reader [23], [14], [12]. Since the frame-size of the BFSA is fixed, its implementation is simplistic. However, the system's efficiency drops significantly in the event of there being too large or too small tag counts. For instance, no tag may be identified in a read cycle if there are too many tags within the interrogation zone. On the other hand, under small tag counts where large frame-size is used, lots of empty slots are produced, resulting in decreased system efficiency.

In ALOHA-based anti-collision, there are three kinds of slot as shown in Figure 1: 1) Empty slot where there is no tag reply; 2) Successful slot where there is only one tag reply (Tag 4); and 3) Collision slot where there is more than one tag reply (Tag 2 and Tag 3 ).

\subsection{Dynamic Framed-Slotted ALOHA Algorithm}

The Dynamic Framed-Slotted ALOHA (DFSA) overcomes the problems associated with BFSA by dynamically changing the frame-size according to estimated number of Backlog, which is a number of tags that have not been read. In DFSA, each tag in an interrogation zone selects one of the given $N$ slots to transmit its identifier, and all tags will be recognised after a few frames. Each frame is formed of specific number of slots that is used for the communication between the readers and the tags. To determine the frame-size, it gathers and uses information such as number of successful slots, empty slots, and collision slots from the previous round to predict the appropriate frame-size for the next identification round [6], [14], [5], [25].

DFSA can identify the tag efficiently because the reader adjusts the frame-size according to the estimated number of tags. However, the frame-size change alone cannot reduce sufficiently the tag collision when there are large numbers of tags because it cannot increase the frame-size indefinitely. DFSA has various versions depending on different tag estimation methods used. There have been several researches to improve the accuracy of frame-size by implementing a Frame Estimation Tool [13], [11], [3].

\subsection{Enhanced Dynamic Framed-Slotted ALOHA Algorithm}

The DFSA algorithms change the frame-size to increase the performance efficiency of the tag identification. However, as the number of tags becomes larger than the frame-size, the probability of collision increases rapidly. If number of unread tags can be estimated accurately, frame-size can be determined to maximise the system efficiency or minimise the tag collision probability. For instance, when the number of tags is large, the probability of tag collision can be reduced by increasing the frame-size. However, the frame-size cannot be increased indefinitely. When the number of unread tags is too large to achieve high system efficiency, the number of responding tags somehow must be restricted so that the 
optimal number of tags responds to the given frame-size [14], [13].

The Enhanced Dynamic Framed-Slotted ALOHA (EDFSA) first estimates the number of unread tags. If the number of tags within the interrogation zone is larger than the maximum frame-size, the EDFSA algorithm splits the number of Backlog into number of groups and allows only one group of tags to respond. When the reader limits the number of responding tags, it transmits the number of tag sets and a random number to the tags when it issues the query. Only the tag that picks zero as its slot counter responds to the request. If the number of estimated Backlog is below the threshold, the reader adjusts the frame-size without grouping the unread tags. After each read cycle, the reader estimates the number of unread tags and adjusts its frame-size. This procedure repeats until all the tags are read [14], [13]. The problem with EDFSA method is, it assumes that 256 is the optimal frame-size and splits tags into group set by using the power of two $(2,4,8 \ldots)$. This results in a decrease in system efficiency when the number of tags is a fraction above the threshold and the number of group sets will be doubled.

\subsection{Backlog Estimation Techniques}

In order to predict accurate number of unread tags and determine the new frame-size, ALOHA-based anticollision algorithms gather and use information such as number of successful slots, empty slots, and collision slots from the previous round to predict the appropriate frame-size for the next identification round. In past literature, there have been several methodologies related to Backlog estimation. These approaches include Schoute method [22], Vogt method [24], Bayesian method [9], Chen1 and Chen2 methods [2], and PTES method [18]. Unfortunately, some of these methodologies either have low performance efficiencies or are too complicated to be implemented for some RFID applications. These methods are explained as follows:

\subsubsection{Schoute Backlog Estimation Technique}

Schoute [22] developed a Backlog estimation technique for Dynamic Framed-Slotted ALOHA using Poisson distribution. The Backlog, after the current frame Bt, is given by equation:

$B t=2.39 \times c$

Where $c$ represents the number of collided slot in the current frame.
Schoute method is the simplest, easy to implement with low overhead computation, and provides accurate tag estimation [27].

\subsubsection{Vogt Estimation Techniques}

In [24], a procedure to estimate Backlog is presented by minimising the difference between the observed value, including number of empty slot $h$, successful slot $s$, and collision slot $c$, and the expected value $\mathrm{E}(\mathrm{H}), \mathrm{E}(\mathrm{S})$, $\mathrm{E}(\mathrm{C})$. In order to find the comparative precise Back$\log$, the reader needs to resolve the equation below:

$\min \left(\begin{array}{c}h \\ s \\ c\end{array}\right)-\left(\begin{array}{c}E_{N}(H) \\ E_{N}(S) \\ E_{N}(C)\end{array}\right)$

Vogt method presents the most accurate tag estimation, however, the complexity of the algorithm resulted in high overhead and therefore cannot be applied to Gen2 protocol [12].

\subsubsection{Bayesian Estimation Techniques}

Bayesian method [9] first computes the frame size L based on the current probability distribution of the random variable $\mathrm{N}$ that represents the number of tags transmitting. Then it starts frame with $\mathrm{L}$ slots and waits for tag replies and update probability distribution of $\mathrm{N}$, based on evidence from the reader at the end of the frame. The evidence comprises the number of empty slots, successful slots, and collision slots in the last frame. The method then adjusts probability distribution $\mathrm{N}$ by considering newly arriving tags and departing tags including the ones which successfully replied and do not transmit in subsequent slots. Bayesian method requires the most complex computation and implementation of algorithm [15]. This resulted in high overhead and delays the identification process. It is therefore not widely applied in RFID systems.

\subsubsection{Chen1 and Chen2 Estimation Techniques}

Most of the static algorithms estimate the Backlog with the number of collided slot. However, Chen1 method [2] estimates the Backlog, based on the empty slot information, through the probability of finding $h$ empty slots after completing a frame $L$. Chen2 method [2] is a simpler way to estimate the number of tags, which is illustrated as follows:

$$
n=(L-1) \times \frac{s}{h}
$$


If $\mathrm{h}=0, \mathrm{n}$ is set to a certain upper bound for the tags estimate.

Chen1 and Chen2 methods have worse performances than simple Schoute method, according to [26]. Chen1 method also requires complex computation, which leads to high overhead and delay the tag identification process.

\subsubsection{Precise Tag Estimation Scheme}

Precise Tag Estimation Scheme (PTES) [18] approach assumes that for the current identification round, each collision slot has at least 2 tags colliding. However, it is unknown how many tags actually caused the collision. There is exactly 1 tag per successful slot, thus successful slots are not taken into consideration. On the other hand, empty slots will continuously occur during the next rounds of identification despite the frame-size. Therefore, Backlog after the current frame is defined by equation:

Backlog $=\left\lceil\left(V_{1} \times c+V_{2} \times e\right)\right\rceil$

Where $c$ is Collision Slot; $e$ is Empty slot; $V_{1}$ and $\mathrm{V}_{2}$ are variables for collision slot and empty prediction respectively.

In this study, we have chosen PTES approach to be used as Backlog estimation tool in our newly proposed Probabilistic Cluster-Based Technique. PTES method is easy to implement with low overhead computation and provides accurate tag estimation. This approach has the best performance in terms of system efficiency, and the number of frames and slots queried by the algorithm. The optimal efficiency of PTES can be obtained when the suitable parameters have been chosen for the specified initial frame-size.

\section{Probabilistic Cluster-Based Technique}

To address shortcomings of the current probabilistic anti-collision methods, we propose a Probabilistic ClusterBased Technique (PCT) to minimise identification time and optimise performance efficiency. The Precise Tag Estimation Scheme (PTES) [18] is also employed as accurate frame-size prediction for PCT.

This section explains the fundamental of probabilistic anti-collision, the PTES frame-size estimation method, and the proposed PCT rules.

\subsection{The Fundamentals for Probabilistic Tag} Estimation

In probabilistic anti-collision schemes, to estimate the number of present tags, Binomial distribution has been utilised [26], [15], [7], [8]. For a given initial $Q$ in a frame with $F$ slots and $n$ tags, the expected value of the number of slots with occupancy number $x$ is as follows:

$$
a_{x}=n \times C_{n}^{x}\left(\frac{1}{F}\right)^{x}\left(1-\frac{1}{F}\right)^{n-x}
$$

Therefore, the expected number of Empty slot $e$, Successful slot $s$, and Collision slot $c$ are given by the following equations:

$$
\left\{\begin{array}{l}
e=a_{0}=F\left(1-\frac{1}{F}\right)^{n} \\
s=a_{1}=n\left(1-\frac{1}{F}\right)^{n-1} \\
c=a_{k}=F-a_{0}-a_{1}
\end{array}\right.
$$

Thus, the system efficiency (E) is defined as the ratio between the number of Successful slot and the frame-size given by the following equations:

$$
E=\frac{s}{F}=\frac{n\left(1-\frac{1}{F}\right)^{n-1}}{F}=n \frac{1}{F}\left(1-\frac{1}{F}\right)^{n-1}
$$

It has been proven that the highest efficiency can be obtained if the frame-size $F$ is equal to the number of tags $n$, provided that all slots have the same fixed length [26]:

$$
F(\text { optimal })=n
$$

\subsection{PTES Backlog Estimation}

We have chosen PTES backlog estimation to incorporate with our proposed PCT method. The suggested threshold for frame-size for PTES is set according to estimated number of tags utilising Binomial distribution. For example, if estimated number of tags is around 60 tags, the suggested frame-size would have a $Q$ value of 6 . The frame-size, where $\mathrm{Q}=6$, will allow a minimum number of 33 tags and a maximum number of 64 tags to be identified.

Table 1 shows Minimum and Maximum number of tags allowed per suggested frame-size, and Minimum and Maximum boundary of random numbers generated per frame-size. Maximum number of tags allowed in each frame-size is calculated by $2^{Q}$ and minimum number of tags allowed is calculated by $2^{Q-1}+1$. The 
Table 1 Suggested frame-size boundary (B) and minimum and maximum number of tags (NT) for specific estimated number of tags.

\begin{tabular}{|c|c|c|c|c|c|c|c|c|c|c|}
\hline & \multicolumn{2}{|c|}{$\mathrm{Q}=\mathbf{1}$} & \multicolumn{2}{|c|}{$\mathrm{Q}=2$} & \multicolumn{2}{|c|}{$\mathrm{Q}=\mathbf{3}$} & \multicolumn{2}{|c|}{$Q=4$} & \multicolumn{2}{|c|}{$\mathrm{Q}=5$} \\
\hline & NT & B & $\mathbf{N T}$ & B & $\mathbf{N T}$ & B & NT & B & NT & B \\
\hline Min & 1 & 0 & 3 & 0 & 5 & 0 & 9 & 0 & 17 & 0 \\
\hline \multirow[t]{3}{*}{ Max } & 2 & 1 & 4 & 3 & 8 & 7 & 16 & 15 & 32 & 31 \\
\hline & \multicolumn{2}{|c|}{$\mathrm{Q}=6$} & \multicolumn{2}{|c|}{$\mathrm{Q}=7$} & \multicolumn{2}{|c|}{$\mathrm{Q}=\mathbf{8}$} & \multicolumn{2}{|c|}{$\mathrm{Q}=\mathbf{9}$} & \multicolumn{2}{|c|}{$\mathrm{Q}=10$} \\
\hline & NT & B & NT & B & NT & B & $\mathbf{N T}$ & B & NT & B \\
\hline Min & 33 & 0 & 65 & 0 & 129 & 0 & 257 & 0 & 513 & 0 \\
\hline \multirow[t]{3}{*}{ Max } & 64 & 63 & 128 & 127 & 256 & 255 & 512 & 511 & 1024 & 1023 \\
\hline & \multicolumn{2}{|c|}{$\mathrm{Q}=11$} & \multicolumn{2}{|c|}{$\mathrm{Q}=12$} & \multicolumn{2}{|c|}{$\mathrm{Q}=13$} & \multicolumn{2}{|c|}{$\mathrm{Q}=14$} & \multicolumn{2}{|c|}{$\mathrm{Q}=15$} \\
\hline & $\mathbf{N T}$ & $\bar{B}$ & $\mathbf{N T}$ & $\bar{B}$ & $\mathbf{N T}$ & $\bar{B}$ & $\mathbf{N T}$ & $\bar{B}$ & $\mathbf{N T}$ & $\bar{B}$ \\
\hline Min & 1025 & 0 & 2049 & 0 & 4097 & 0 & 8193 & 0 & 16385 & 0 \\
\hline Max & 2048 & 2047 & 4096 & 4095 & 8192 & 8191 & 16384 & 16383 & 32768 & 32767 \\
\hline
\end{tabular}

maximum frame-size boundary is calculated by $2^{Q}-1$, while the minimum frame-size boundary is always 0 . The table only demonstrates up to $\mathrm{Q}=15$.

Algorithm 1 demonstrates the PTES algorithm applied for the number of backlog estimation, and either keep the current frame-size or readjust the frame-size for the next identification cycle.

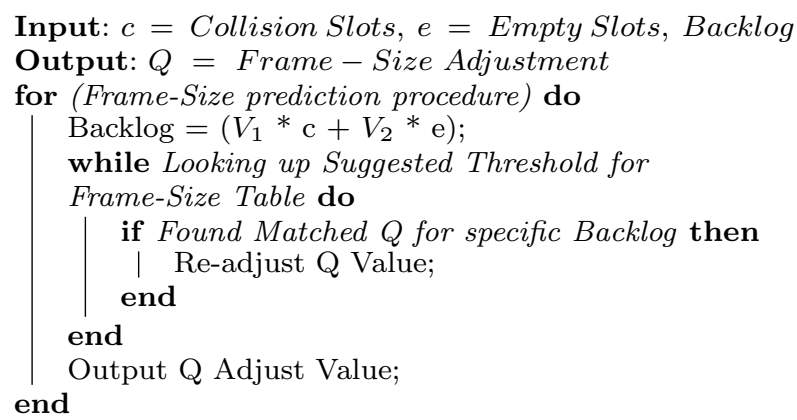

Algorithm 1: PTES Algorithm

\subsection{PCT Anti-Collision Approach}

The PCT method employed a dynamic probabilistic algorithm concept and uses group splitting rule to split Backlog into group if the number of unread tags is higher than the maximum frame-size.

PCT approach first estimates the number of Back$\log$. If the number of Backlog within the interrogation zone is larger than the specific frame-size, it splits the number of Backlog into a number of groups and allows only one group of tags to respond. The reader then issues "Query", which contains a 'Q' parameter to specify the frame-size. Each selected tag in the group will pick a random number between 0 to $2^{Q}-1$ and put it into its slot counter. Only the tag that picks zero as its slot counter responds to the request. When the number of estimated Backlog is below the threshold, the reader adjusts the frame-size without grouping the unread tags. After each identification cycle, the reader estimates the number of Backlog using PTES algorithm and adjust its frame-size.

\subsubsection{Probabilistic Anti-Collision Algorithm using PTES}

PCT approach first estimates the number of unread tags, then it decided if the number of tags needs to be spliced or not. The probabilistic anti-collision algorithm, using PTES as accurate frame-size prediction, is then applied to each selected group of tag.

Algorithm 2 demonstrates our probabilistic based anti-collision algorithm applied to each selected group of tags, where only one group of tags respond to the reader. There are three kinds of slot:

1. Successful slot: Where there is only one tag reply, the reader sends $\mathrm{ACK}(\mathrm{RN} 16)$ to a tag. The tag then backscatter its EPC to the reader and the reader issues QueryRep for the next slot.

2. Empty slot: Where there is no tag reply, the reader then issues QueryRep for the next slot.

3. Collision slot: Where there is more than one tag reply, the reader then issues QueryRep for the next slot.

After "QueryRep" command is received, each tag decreases its slot counter by 1 . At the end of each frame, the reader checks if all tags have been identified, estimates the number of Backlog using PTES algorithm, and adjusts its frame-size.

\subsubsection{PCT Preliminary}

Instead of splitting tags into group randomly, the PCT approach derived new rules using particular equations 
Reader sends Query

for (Identification procedure) do

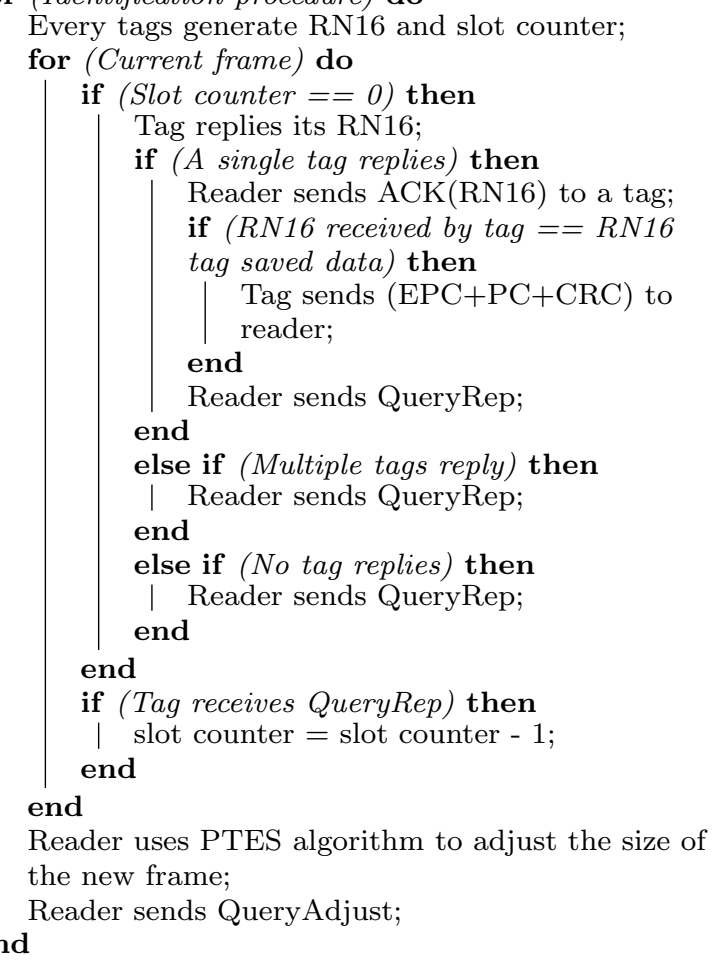

Algorithm 2: Probabilistic anti-collision algorithm with PTES Frame-Size Prediction

according to the optimal system efficiency obtained for specific number of tags. We first conducted an experiment to acquire optimal frame-size for specific number of tags as shown in Figure 2. It can be seen that the optimal system efficiency achieved by the probabilistic method is approximately $38 \%$ and the optimal number of tags are close to the maximum frame-size. Efficiency is calculated as shown in Equation 1:

$$
\text { Efficiency }=\left(\frac{S}{S+C+E}\right)
$$

Where $\mathrm{S}$ is the number of Successful slots, $\mathrm{C}$ is the number of Collision slots, and $\mathrm{E}$ is the number of Empty slots.

From the results acquired for performance efficiency evaluation, we have developed five cluster-based equations to find a minimum and maximum number of tags suitable for particular frame-size. These minimum and maximum number of tags are derived to acquire the optimal performance efficiencies as in Figure 2. Each equation is then used to exploit rules for PCT.

To show the derivation of all five cluster-based equations in detailed, Table 2 demonstrated given information found from Figure 2, and all missing fields. From table 2 , it is visible that at optimal system efficiency of

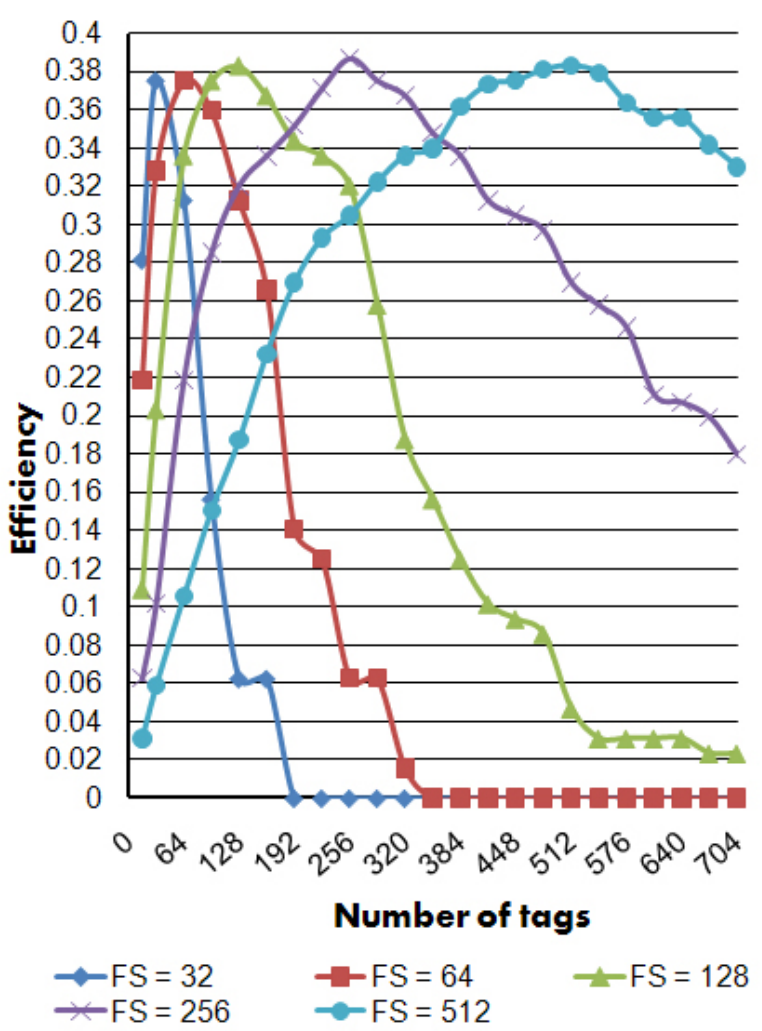

Fig. 2 Performance efficiency of different frame-size on different number of tags

$38 \%$, the number of tags is equal to the available framesize calculated by $2^{Q}$. We have set the minimum and maximum boundary efficiencies at $33 \%$. The information on maximum number of tags at $33 \%$ is also available from Figure 2. For example, when $\mathrm{Q}$ is equal to 8 , the optimal percentage efficiency can be obtained at 256 tags and the number of tags at maximum boundary is equal to 352 tags.

Table 2 Available Information and Missing fields on System Efficiency. MinB = Minimum point of occurrence, MaxB $=$ Maximum point of occurrence, Opt. $=$ Optimal point of occurrence

\begin{tabular}{|c|c|c|c|}
\hline Q & MinB at 33\% & MaxB at 33\% & Opt. at 38\% \\
\hline 9 & Unknown & 704 & 512 \\
8 & Unknown & 352 & 256 \\
7 & Unknown & 176 & 128 \\
6 & Unknown & 88 & 64 \\
5 & Unknown & 44 & 32 \\
4 & Unknown & Unknown & 16 \\
3 & Unknown & Unknown & 8 \\
2 & Unknown & Unknown & 4 \\
1 & Unknown & Unknown & 2 \\
\hline
\end{tabular}

Table 3 demonstrated the derived answers for missing fields from Table 2. The minimum boundary with 
$33 \%$ efficiency is calculated by the maximum boundary of the previous frame plus 1. Thus, for Q8, the minimum boundary is equal to $177(176+1)$. After finding all information needed, the reverse engineered equations for maximum and minimum boundaries are derived for each Q. After we found all outcomes for each Q, it is now possible to find the reverse engineered equation for two or more type of Qs. For example, if Q8 and Q7 is applicable, then the reversed engineered equation for maximum boundary is equal to $\left[2^{Q}+2^{(Q-1)}-2^{(Q-2)}\right.$ $\left.+2^{(Q-3)}\right]+\left[2^{(Q-1)}+2^{(Q-2)}-2^{(Q-3)}+2^{(Q-4)}\right]$.

Table 3 Derived Equations for Missing fields on System Efficiency. $\mathrm{MinB}=$ Minimum point of occurrence, $\mathrm{MaxB}=$ Maximum point of occurrence

\begin{tabular}{|c|c|c|}
\hline Q & $\begin{array}{c}\text { MinB } \\
\mathbf{3 3 \%}\end{array}$ & $\begin{array}{c}\text { Reverse Engineered } \\
\text { Equation for MinB }\end{array}$ \\
\hline 9 & 353 & $\left(2^{(Q-1)}+2^{(Q-2)}-2^{(Q-3)}+2^{(Q-4)}\right)+1$ \\
8 & 177 & $\left(2^{(Q-1)}+2^{(Q-2)}-2^{(Q-3)}+2^{(Q-4)}\right)+1$ \\
7 & 89 & $\left.2^{(Q-1)}+2^{(Q-2)}-2^{(Q-3)}+2^{(Q-4)}\right)+1$ \\
6 & 45 & $\left(2^{(Q-1)}+2^{(Q-2)}-2^{(Q-3)}+2^{(Q-4)}\right)+1$ \\
5 & 23 & $\left.2^{(Q-1)}+2^{(Q-2)}-2^{(Q-3)}+2^{(Q-4)}\right)+1$ \\
4 & 12 & $\left(2^{(Q-1)}+2^{(Q-2)}-2^{(Q-3)}+2^{(Q-4)}\right)+1$ \\
3 & 6 & $\left(2^{(Q-1)}+2^{(Q-2)}-2^{(Q-3)}+2^{(Q-4)}\right)+1$ \\
2 & 4 & $\left(2^{(Q-1)}+2^{(Q-2)}-2^{(Q-3)}+2^{(Q-4)}\right)+1$ \\
1 & 2 & $\left(2^{(Q-1)}+2^{(Q-2)}-2^{(Q-3)}+2^{(Q-4)}\right)+1$ \\
\hline \multicolumn{3}{|c|}{} \\
\hline \multicolumn{3}{|c|}{ Reverse Engineered } \\
$\mathbf{Q}$ & $\mathbf{M a x B}$ & $\mathbf{E q u a t i o n}$ for MaxB \\
\hline 9 & $\mathbf{3 3 \%}$ & $2^{Q}+2^{(Q-1)}-2^{(Q-2)}+2^{(Q-3)}$ \\
8 & 354 & $2^{Q}+2^{(Q-1)}-2^{(Q-2)}+2^{(Q-3)}$ \\
7 & 176 & $2^{Q}+2^{(Q-1)}-2^{(Q-2)}+2^{(Q-3)}$ \\
6 & 88 & $2^{Q}+2^{(Q-1)}-2^{(Q-2)}+2^{(Q-3)}$ \\
5 & 44 & $2^{Q}+2^{(Q-1)}-2^{(Q-2)}+2^{(Q-3)}$ \\
4 & 22 & $2^{Q}+2^{(Q-1)}-2^{(Q-2)}+2^{(Q-3)}$ \\
3 & 11 & $2^{Q}+2^{(Q-1)}-2^{(Q-2)}+2^{(Q-3)}$ \\
2 & 5 & $2^{Q}+2^{(Q-1)}-2^{(Q-2)}+2^{(Q-3)}$ \\
1 & 3 & $2^{Q}+2^{(Q-1)}-2^{(Q-2)}+2^{(Q-3)}$ \\
\hline
\end{tabular}

$\beta=2^{Q}+2^{(Q-1)}-2^{(Q-2)}+2^{(Q-3)}$

$\kappa=2^{(Q-1)}+2^{(Q-2)}-2^{(Q-3)}+2^{(Q-4)}$

$\mu=2^{(Q-2)}+2^{(Q-3)}-2^{(Q-4)}+2^{(Q-5)}$

In order to simplify the derived equations, we employ the use of $\beta$ (Beta), $\kappa$ (Kappa), and $\mu(\mathrm{Mu})$, and assigned these three icons to express each rule. In this research, we proposed three rules for PCT: PCT256, PCT128, and PCT-E (PCT-Extended). All rules split the number of Backlog into groups then used one of initial Q8 (frame-size 256), Q7 (frame-size 128), or Q6 (frame-size 64), to identify a current set of tags. Equation 2 shows the conversion of all three key sets, for the five cluster-based equations, into $\beta, \kappa$, and $\mu$.

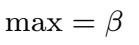

$\max =\beta+\mu$

$\min =\beta+1$

$\max =\beta+\kappa$

$\min =\beta+1$

$\max =\beta+\kappa$

$\min =[\beta+\mu]+1$

$\max =\beta+\kappa+\mu$

$\min =[\beta+\kappa]+1$

For equations 3, 4, 5, 6 and 7, we applied three key sets within these equations. These key sets employed $\beta, \kappa$, and $\mu$ and applied them into each PCT rule, as shown in Table 4.

Table 4 displays equations 3 to 7 , with the minimum and maximum bounds for each rule. For instance, Equation 3 is applied to all three rules: PCT256, PCT128, and PCT-E. Equation 4, however, only apply to PCTE.

Table 4 The conversion of PCT rules to $\beta$ Beta, $\kappa$ Kappa, and $\mu \mathrm{Mu}$

\begin{tabular}{|c|c|c|c|}
\hline & PCT256 & PCT128 & PCT-E \\
\hline $\begin{array}{l}\text { Max } \\
\text { Min }\end{array}$ & \multicolumn{3}{|c|}{$\begin{array}{c}\beta \\
\kappa+1(\mathbf{3})\end{array}$} \\
\hline \multirow{3}{*}{$\begin{array}{l}\text { Max } \\
\text { Min }\end{array}$} & \multirow{3}{*}{\multicolumn{2}{|c|}{$\begin{array}{c}\beta+\kappa \\
\beta+1(5)\end{array}$}} & $\begin{array}{c}\beta+\mu \\
\beta+1(4)\end{array}$ \\
\hline & & & $\begin{array}{c}\beta+\kappa \\
{[\beta+\mu]+1(6)}\end{array}$ \\
\hline & & & $\begin{array}{c}\beta+\kappa+\mu \\
{[\beta+\kappa]+1(7)}\end{array}$ \\
\hline
\end{tabular}

\subsubsection{PCT Rules}

PCT approach derived new rules using particular equations expressed by $\beta$ Beta, $\kappa$ Kappa, and $\mu \mathrm{Mu}$. All rules split the number of Backlog into groups then used one of Q8 (frame-size 256), Q7 (frame-size 128), or Q6 (framesize 64$)$, to identify a current set of tags. We make the assumption that the performance efficiency can be improved by dividing tags into accurate number of groups, 
then perform the tag identification separately for each group. In this research, we have chosen the frame-size of 256, 128 and 64 for our PCT rules because the initial $\mathrm{Q}$ of 8,7 and 6 provides the most appropriate range for current RFID reader and passive tags specification. Generally, the Ultra-High Frequency (UHF) reader is capable of capturing various number of passive RFID tags that depends on the reader type and tag class (e.g. Class 0: Read-only tag). Thus, selected initial Qs are the most suitable for our proposed rules. Each PCT rule, with the minimum and maximum bounds, is explained as follows:

PCT256 PCT256 uses either frame-size of $256(\mathrm{Q}=$ $8)$ or frame-size of $128(\mathrm{Q}=7)$ for tag identification. We assume that the identification time and performance efficiency of our proposed PCT256 will advance from the existing probabilistic approaches. From the preliminary of all PCT rules, we obtained specific equations to calculate minimum and maximum bounds for the PCT256 rule. These equations are applied as shown in Table 5.

Table 5 PCT256 Boundary Computation - number of group (Frame-Size 256 and 128), and minimum and maximum bounds

\begin{tabular}{|c|c|c|c|}
\hline \multicolumn{4}{|c|}{ PCT256 Boundary Computation } \\
\hline F256 & F128 & MinBound & MaxBound \\
\hline$\ldots$ & $\ldots$ & $\ldots$ & $\ldots$. \\
4 & - & {$[3 \beta+\kappa]+1$} & $4 \beta$ \\
3 & 1 & $3 \beta+1$ & $3 \beta+\kappa$ \\
3 & - & {$[2 \beta+\kappa]+1$} & $3 \beta$ \\
2 & 1 & $2 \beta+1$ & $2 \beta+\kappa$ \\
2 & - & {$[\beta+\kappa]+1$} & $2 \beta$ \\
1 & 1 & $\beta+1$ & $\beta+\kappa$ \\
1 & - & $\kappa+1$ & $\beta$ \\
$\ldots$ & $\ldots$ & $\ldots$ & $\ldots$ \\
\hline
\end{tabular}

Table 5 displays relevant equations for minimum and maximum bounds calculation for the PCT256 rule. From the table, we can see that there are two framesize, 256 and 128, for grouping division. For example, the minimum bound is calculated by $3 \beta+1$ when the number of group division comprised 3 groups of 256 and 1 group of 128 , and the maximum bound is calculated by $3 \beta+\kappa$. Following the computation, the minimum and maximum bounds are 1057 and 1232 respectively, as shown in Table 6 .

After applying specific equations for each group division, Table 6 shows the final PCT rule for PCT256. For instance, if the number of Backlog equals to 900 tags, the PCT256 algorithm will split the unread tags into 3 groups of Q8 (256).

Algorithm 3 demonstrates the group splitting algorithm using PCT256 rule, which either keep tag in a
Table 6 PCT256 Rule - The number of unread tags (Back$\log$ ), optimal frame-size (FA and FB), and number of group (GA and GB)

\begin{tabular}{|c|c|c|c|c|}
\hline \multicolumn{5}{|c|}{ PCT256 Rule } \\
\hline Backlog & FA & GA & FB & GB \\
\hline$\ldots$. & $\ldots$. & $\ldots$. & $\ldots$. & $\ldots$ \\
1233 to 1408 & 256 & 4 & - & - \\
1057 to 1232 & 256 & 3 & 128 & 1 \\
881 to 1056 & 256 & 3 & - & - \\
705 to 880 & 256 & 2 & 128 & 1 \\
529 to 704 & 256 & 2 & - & - \\
353 to 528 & 256 & 1 & 128 & 1 \\
177 to 352 & 256 & 1 & - & - \\
89 to 176 & 128 & 1 & - & - \\
45 to 88 & 64 & 1 & - & - \\
23 to 44 & 32 & 1 & - & - \\
12 to 22 & 16 & 1 & - & - \\
6 to 11 & 8 & 1 & - & - \\
$\ldots .$. & $\ldots$. & $\ldots$. & $\ldots$. & $\ldots$ \\
\hline
\end{tabular}

single group or split tag into number of groups according to PCT256 rule.

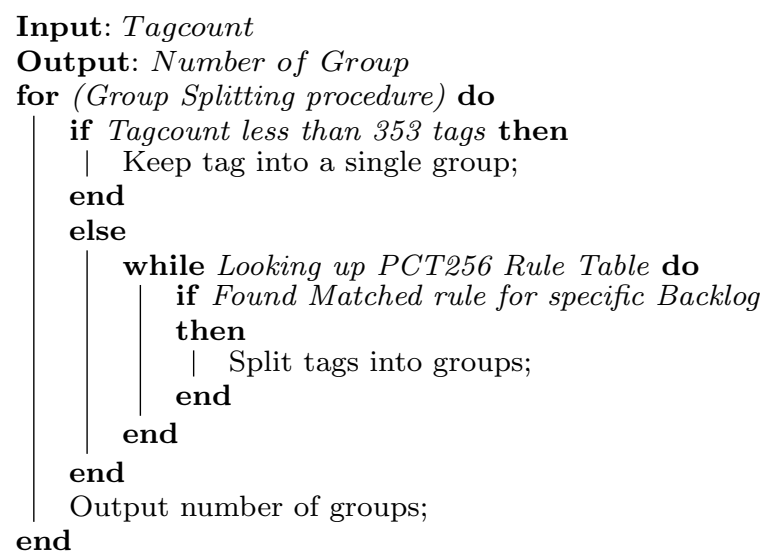

Algorithm 3: Group Splitting Algorithm using PCT256 Rule

PCT128 PCT128 uses either frame-size of 128 (Q $=7)$ or frame-size of $64(\mathrm{Q}=6)$ for tag identification. The PCT128 contains higher number of groups in some cases, compared with the PCT256 method, which may result in worse performance efficiency for specific number of tags. We calculate minimum and maximum bounds for the PCT128 rule according to specific equations. These equations are applied as shown in Table 7.

Table 7 displays the relevant equations for minimum and maximum bounds calculation for the PCT128 rule. From the table, it can be seen that there are two framesize, 128 and 64, for grouping division. For example, the minimum bound is calculated by $5 \beta+1$ when the number of group division comprises 5 groups of 128 and 
Table 7 PCT128 Boundary Computation - number of group (Frame-Size 128 and 64), and minimum and maximum bounds

\begin{tabular}{|c|c|c|c|}
\hline \multicolumn{4}{|c|}{ PCT128 Boundary Computation } \\
\hline F128 & F64 & MinBound & MaxBound \\
\hline$\ldots$. & $\cdots$ & $\ldots$ & $\ldots$ \\
8 & - & {$[7 \beta+\kappa]+1$} & $8 \beta$ \\
7 & 1 & $7 \beta+1$ & $7 \beta+\kappa$ \\
7 & - & {$[6 \beta+\kappa]+1$} & $7 \beta$ \\
6 & 1 & $6 \beta+1$ & $6 \beta+\kappa$ \\
6 & - & {$[5 \beta+\kappa]+1$} & $6 \beta$ \\
5 & 1 & $5 \beta+1$ & $5 \beta+\kappa$ \\
5 & - & {$[4 \beta+\kappa]+1$} & $5 \beta$ \\
4 & 1 & $4 \beta+1$ & $4 \beta+\kappa$ \\
4 & - & {$[3 \beta+\kappa]+1$} & $4 \beta$ \\
3 & 1 & $3 \beta+1$ & $3 \beta+\kappa$ \\
3 & - & {$[2 \beta+\kappa]+1$} & $3 \beta$ \\
2 & 1 & $2 \beta+1$ & $2 \beta+\kappa$ \\
2 & - & {$[\beta+\kappa]+1$} & $2 \beta$ \\
1 & 1 & $\beta+1$ & $\beta+\kappa$ \\
1 & - & $\kappa+1$ & $\beta$ \\
$\ldots$. & $\ldots$. & $\ldots$. & $\ldots$ \\
\hline
\end{tabular}

1 group of 64 , and the maximum bound is calculated by $5 \beta+\kappa$. Following the computation, the minimum and maximum bounds are 881 and 968 respectively, as shown in Table 8.

Table 8 PCT128 Rule - The number of unread tags (Back$\log$ ), optimal frame-size (FA and FB), and number of group (GA and GB)

\begin{tabular}{|c|c|c|c|c|}
\hline \multicolumn{5}{|c|}{ PCT128 Rule } \\
\hline Backlog & FA & GA & FB & GB \\
\hline$\ldots$. & $\ldots$. & $\ldots$ & $\ldots$. & $\ldots$. \\
1321 to 1408 & 128 & 8 & - & - \\
1233 to 1320 & 128 & 7 & 64 & 1 \\
1145 to 1232 & 128 & 7 & - & - \\
1057 to 1144 & 128 & 6 & 64 & 1 \\
969 to 1056 & 128 & 6 & - & - \\
881 to 968 & 128 & 5 & 64 & 1 \\
793 to 880 & 128 & 5 & - & - \\
705 to 792 & 128 & 4 & 64 & 1 \\
617 to 704 & 128 & 4 & - & - \\
529 to 616 & 128 & 3 & 64 & 1 \\
441 to 528 & 128 & 3 & - & - \\
353 to 440 & 128 & 2 & 64 & 1 \\
265 to 352 & 128 & 2 & - & - \\
177 to 264 & 128 & 1 & 64 & 1 \\
89 to 176 & 128 & 1 & - & - \\
45 to 88 & 64 & 1 & - & - \\
23 to 44 & 32 & 1 & - & - \\
12 to 22 & 16 & 1 & - & - \\
6 to 11 & 8 & 1 & - & - \\
$\ldots . .$. & $\ldots$. & $\ldots .$. & $\ldots$. & $\ldots$. \\
\hline
\end{tabular}

Table 8 shows the PCT rule for PCT128. For instance, if the number of Backlog equals to 900 tags, the PCT128 algorithm will split the unread tags into 5 groups of Q7 (128) and 1 group of Q6 (64).
Algorithm 4 demonstrates the group splitting algorithm using PCT128 rule, and either keep tag in a single group or split tag into number of groups according to $\mathrm{PCT} 128$ rule.

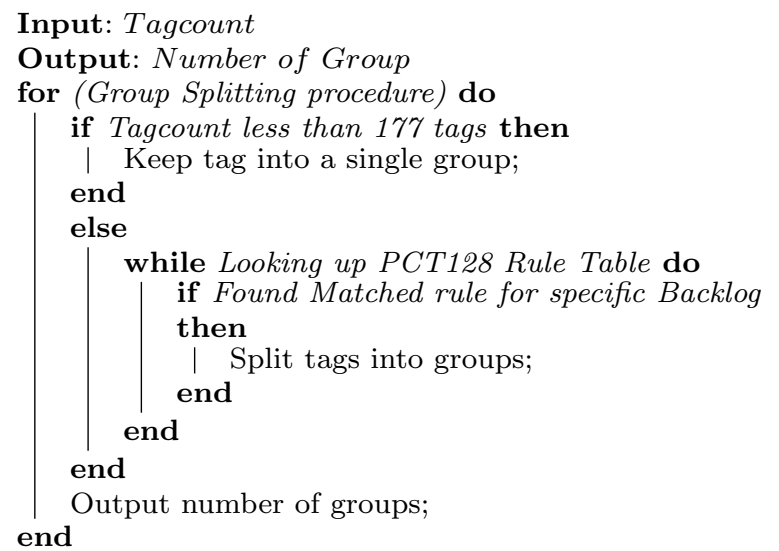

Algorithm 4: Group Splitting Algorithm using PCT128 Rule

PCT-Extended The rules of PCT-Extended (PCTE) are more complex than the PCT256 and PCT128. This is because the PCT-E identify tags using three different frame-size of $256(\mathrm{Q}=8), 128(\mathrm{Q}=7)$, and $64(\mathrm{Q}=6)$ instead of two. We assume that the performance efficiency of PCT-E can improve further from the PCT256. However, the identification time may increase due to the higher number of group applied in each identification round. From the preliminary for all PCT rules, we obtained specific equations to calculate minimum and maximum bounds for the PCT-E rule. These equations are applied as shown in Table 9.

Table 9 PCT-E Boundary Computation - number of group (Frame-Size 256, 128 and 64), and minimum and maximum bounds

\begin{tabular}{|c|c|c|c|c|}
\hline \multicolumn{5}{|c|}{ PCT-E Boundary Computation } \\
\hline F256 & F128 & F64 & MinBound & MaxBound \\
\hline$\ldots$. & $\ldots$ & $\ldots$ & $\ldots$ & $\ldots$ \\
4 & - & - & {$[3 \beta+\kappa+\mu]+1$} & $4 \beta$ \\
3 & 1 & 1 & {$[3 \beta+\kappa]+1$} & $3 \beta+\kappa+\mu$ \\
3 & 1 & - & {$[3 \beta+\mu]+1$} & $3 \beta+\kappa$ \\
3 & - & 1 & $3 \beta+1$ & $3 \beta+\mu$ \\
3 & - & - & {$[2 \beta+\kappa+\mu]+1$} & $3 \beta$ \\
2 & 1 & 1 & {$[2 \beta+\kappa]+1$} & $2 \beta+\kappa+\mu$ \\
2 & 1 & - & {$[2 \beta+\mu]+1$} & $2 \beta+\kappa$ \\
2 & - & 1 & $2 \beta+1$ & $2 \beta+\mu$ \\
2 & - & - & {$[\beta+\kappa+\mu]+1$} & $2 \beta$ \\
1 & 1 & 1 & {$[\beta+\kappa]+1$} & $\beta+\kappa+\mu$ \\
1 & 1 & - & {$[\beta+\mu]+1$} & $\beta+\kappa$ \\
1 & - & 1 & $\beta+1$ & $\beta+\mu$ \\
1 & - & - & $\kappa+1$ & $\beta$ \\
$\ldots$. & $\ldots$ & $\ldots$ & $\ldots$ & $\ldots$ \\
\hline
\end{tabular}


Table 9 presents the relevant equations for minimum and maximum bounds calculation for the PCT-E rule. From the table, it is shown that there are three frame-size, 256, 128 and 64, for grouping division. For instance, the minimum bound is calculated by $[2 \beta+$ $\kappa]+1$ when the number of group division comprised 2 groups of 256, 1 group of 128, and 1 group of 64; and the maximum bound is calculated by $2 \beta+\kappa+\mu$. Following the computation, the maximum and minimum bounds are 881 and 968 respectively, as shown in Table 10.

Table 10 displays the PCT-E rule. For instance, if the number of Backlog equals to 900 tags, the PCT-E algorithm will split the unread tags into 2 groups of Q8 (256), 1 group of Q7 (128), and 1 group of Q6 (64).

Table 10 PCT-E Rule - The Number of Unread Tags (Backlog), Optimal Frame-Size (FA, FB, FC), and Number of Group (GA, GB, GC)

\begin{tabular}{|c|c|c|c|c|c|c|}
\hline \multicolumn{7}{|c|}{ PCT-E Rule } \\
\hline Backlogs & FA & GA & FB & GB & FC & GC \\
\hline$\ldots$. & $\ldots$ & $\ldots$. & $\ldots$ & $\ldots$ & $\ldots$. & $\ldots$ \\
1320 to 1408 & 256 & 4 & - & - & - & - \\
1233 to 1320 & 256 & 3 & 128 & 1 & 64 & 1 \\
1145 to 1232 & 256 & 3 & 128 & 1 & - & - \\
1057 to 1144 & 256 & 3 & - & - & 64 & 1 \\
969 to 1056 & 256 & 3 & - & - & - & - \\
881 to 968 & 256 & 2 & 128 & 1 & 64 & 1 \\
793 to 880 & 256 & 2 & 128 & 1 & - & - \\
705 to 792 & 256 & 2 & - & - & 64 & 1 \\
617 to 704 & 256 & 2 & - & - & - & - \\
529 to 616 & 256 & 1 & 128 & 1 & 64 & 1 \\
441 to 528 & 256 & 1 & 128 & 1 & - & - \\
353 to 440 & 256 & 1 & - & - & 64 & 1 \\
177 to 352 & 256 & 1 & - & - & - & - \\
89 to 176 & 128 & 1 & - & - & - & - \\
45 to 88 & 64 & 1 & - & - & - & - \\
23 to 44 & 32 & 1 & - & - & - & - \\
12 to 22 & 16 & 1 & - & - & - & - \\
6 to 11 & 8 & 1 & - & - & - & - \\
$\ldots$. & $\ldots$. & $\ldots$. & $\ldots$. & $\ldots$. & $\ldots$. & $\ldots$ \\
\hline
\end{tabular}

Algorithm 5 demonstrates the group splitting algorithm using PCT-E rule, and either keep tag in a single group or split tag into a number of groups according to PCT-E rule.

\section{Experimental Evaluation}

In order to show the significance of our proposed methods, we conducted an experimental evaluation and compared our methods to the existing techniques. In this section, we describe the data sets used in the experiment, present the results of the experiment, and provide the analysis.

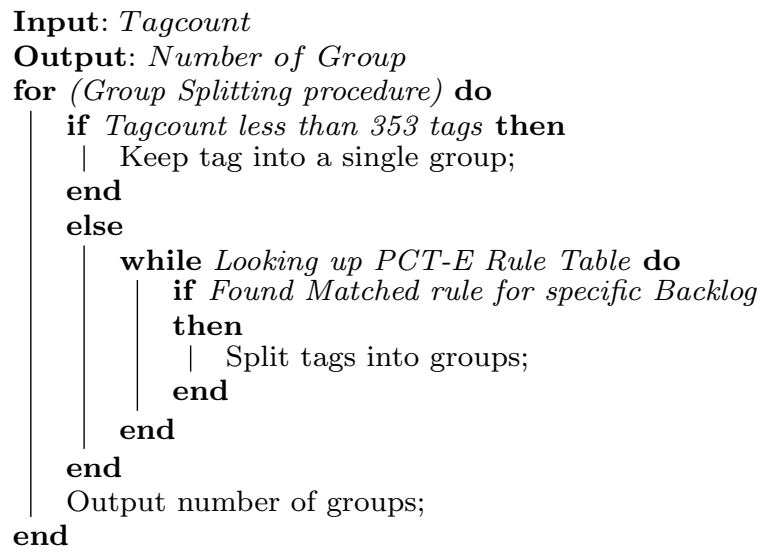

Algorithm 5: Group Splitting Algorithm using PCT-E Rule

\subsection{Data Sets}

The aim of the experiment is to compare the performance of our proposed PCT to the existing probabilistic DFSA and EDFSA anti-collision approaches. The best approach is the one with the highest system efficiency and the lowest number of slots required for tag identification. In this experiment, we considered different number of tags, from 100 to 1400, within the interrogation zone. The number of simulated tags are assumed to be no more than 1400 tags, due to maximum range of UHF reader and passive tags. Before the tag anti-collision algorithm is utilised, the number of tags are unknown.

\subsection{Results}

Our experimentation evaluates the performance of our proposed PCT method to existing DFSA and EDFSA approaches. In Table 11 and Figure 3, it can be seen that both PCT256 and PCT-E produced minimal number of slots during identification process compared to other methods. Specifically, PCT256 and PCT-E technique minimised the number of slots from EDFSA approach when the number of tags is between 400 and 500, and between 800 and 1200 tags. This is because the number of group sets for EDFSA will be doubled when the number of Backlog reached the specific threshold; while PCT increased number of group slowly according to the estimated number of unread tags. As a result, the number of slots are minimised for PCT256. On the other hand, PCT128 performed better than DFSA but does not outperform the EDFSA. According to optimal efficiency displayed in Figure 2, the initial $Q$ of 8 (frame-size $=256$ ) has a wider range of high efficiency compared with the initial $Q$ of 7 . Therefore, PCT256 
with initial frame-size of 256 has a better performance than the PCT128 with initial frame-size of 128.

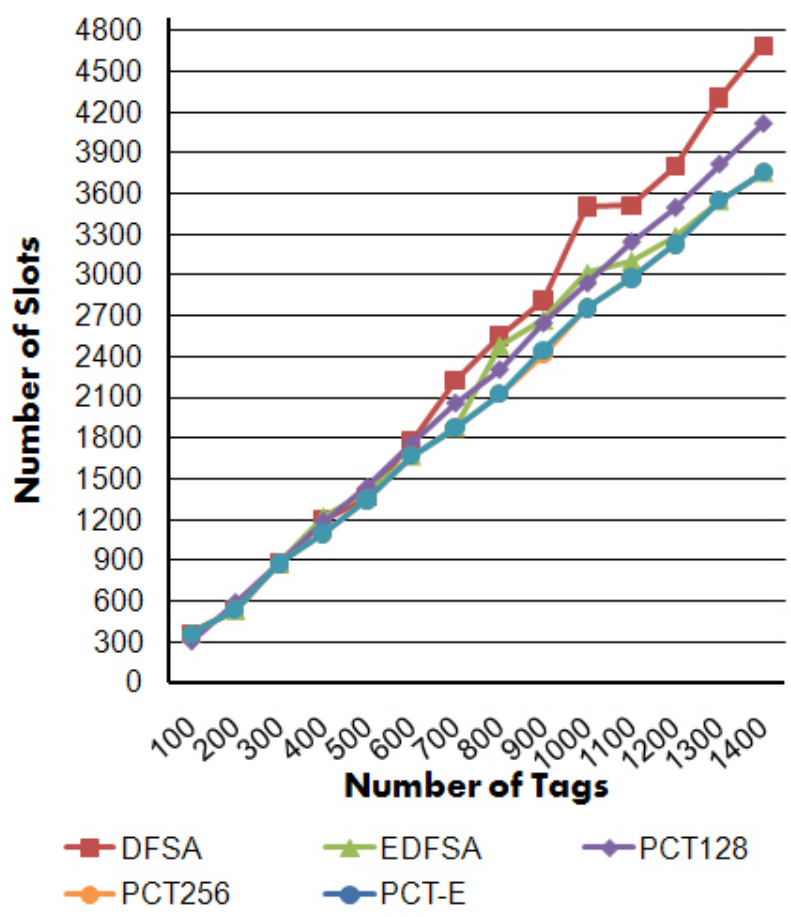

Fig. 3 Number of slots comparison for DFSA, EDFSA, PCT128, and PCT256 methods on different number of tags

Table 11 shows that there is no improvement of our proposed methods compared to existing methods when the number of tags are low (up to around 300 tags). This is because PCT methods start dividing tags into groups only when the number of tags reach the specific threshold. As a result, for certain tag sizes, the number of slots and performance efficiency remained unchanged due to the same identification procedure, compared with DFSA and EDFSA methods. Moreover, Table 11 demonstrates the PCT128 as the only method that has different results when the number of tags are 100 and 200 tags. This is due to the fact that PCT128 is the only method that uses initial frame-size of 128 to predict Backlog. Therefore, even the number of tags still low, the PCT128 start splitting tags into group resulting in different tag outcomes. Furthermore, when the number of tags is 100 tags, PCT128 shows the minimal number of slots issues. The reason for the outcome is because PCT128 uses frame-size of 7 instead of 8 like other methods. Thus, when the number of tags are as low as 100 tags, the PCT128 perform the best. However, we can see from Figure 4 that the performance efficiency of PCT128 stabilised and does not improve any further when the number of tags increased.

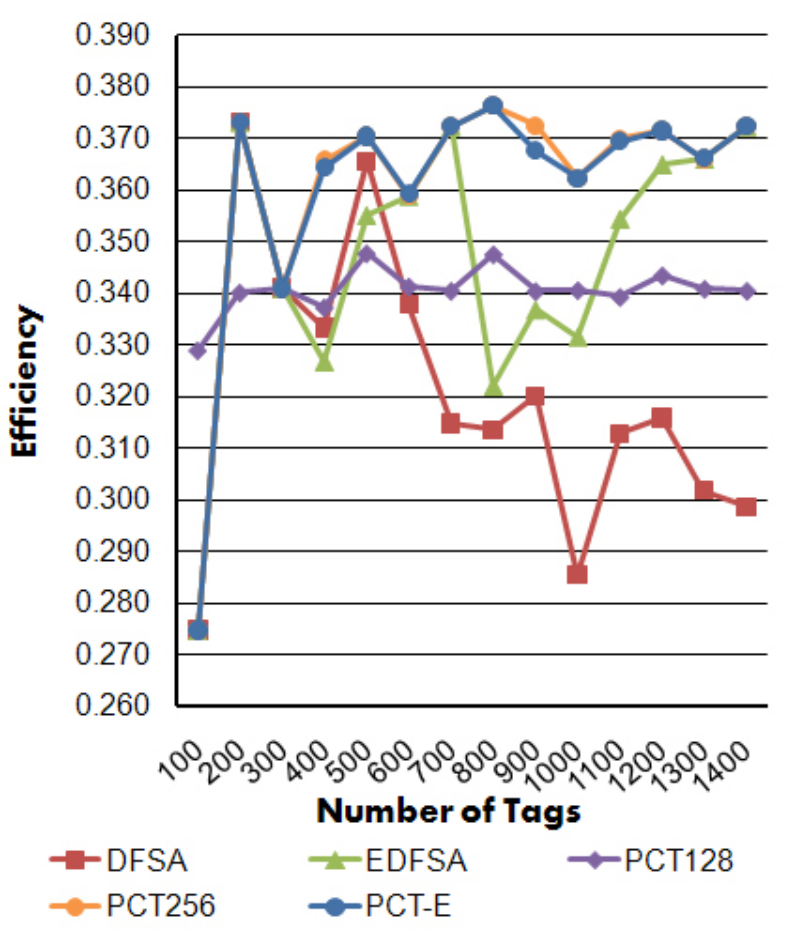

Fig. 4 Performance efficiency for DFSA, EDFSA, PCT128, and PCT256 methods on different number of tags

Table 11 and Figure 4 show that both PCT256 and PCT-E maintained its system efficiency above other methods and has the most stable performance. Nevertheless, the PCT-E required additional number of group sets from the PCT256 method throughout the identification process (see Table 6 and Table 10). As a result, the PCT-E required extra time to initiate a new group compared with the PCT256 method. On the other hand, the DFSA's efficiency dropped dramatically when the number of tags increase, while the EDFSA's efficiency become unstable during the time when number of groups doubled up from 1 to 2 and from 2 to 4 . The PCT128 has steady performance but does not perform as good as PCT256.

Table 12 demonstrates the percentage of improvement of the proposed PCT method versus EDFSA and DFSA methods. It can be seen that when the number of tags are low and the PCT methods have not divided these tags into groups, there is no difference for the outcome of our methods and existing methods, and the percentage of improvement remain unchanged. However, during the time when number of groups doubledup from 1 to 2 (400 tags) and from 2 to 4 (800 tags), PCT256 and PCT-E show the highest percentage of improvement compared with the EDFSA method. The percentage of improvement increased more stably compared with DFSA method, since the DFSA method does not imply group splitting rules. The PCT256 has 
Table 11 Number of slots comparison and Performance efficiency for DFSA, EDFSA, PCT128, PCT256, and PCT-E methods on different number of tags

\begin{tabular}{|c|c|c|c|c|c|c|c|c|c|c|}
\hline \multirow[b]{2}{*}{ Tags } & \multicolumn{5}{|c|}{ Number of Slots } & \multicolumn{5}{|c|}{ Efficiency } \\
\hline & D & ED & P128 & P256 & P-E & $\mathbf{D}$ & ED & P128 & P256 & P-E \\
\hline 100 & 364 & 364 & 304 & 364 & 364 & 0.2747 & 0.2747 & 0.3289 & 0.2747 & 0.2747 \\
\hline 200 & 536 & 536 & 588 & 536 & 536 & 0.3731 & 0.3731 & 0.3401 & 0.3731 & 0.3731 \\
\hline 300 & 880 & 880 & 880 & 880 & 880 & 0.3409 & 0.3409 & 0.3409 & 0.3409 & 0.3409 \\
\hline 400 & 1200 & 1224 & 1186 & 1094 & 1098 & 0.3333 & 0.3268 & 0.3373 & 0.3656 & 0.3643 \\
\hline 500 & 1368 & 1408 & 1438 & 1350 & 1350 & 0.3655 & 0.3551 & 0.3477 & 0.3704 & 0.3704 \\
\hline 600 & 1776 & 1672 & 1758 & 1672 & 1670 & 0.3378 & 0.3589 & 0.3413 & 0.3589 & 0.3593 \\
\hline 700 & 2224 & 1880 & 2056 & 1880 & 1880 & 0.3147 & 0.3723 & 0.3405 & 0.3723 & 0.3723 \\
\hline 800 & 2552 & 2484 & 2302 & 2126 & 2126 & 0.3135 & 0.3221 & 0.3475 & 0.3763 & 0.3763 \\
\hline 900 & 2812 & 2672 & 2644 & 2416 & 2448 & 0.3201 & 0.3368 & 0.3404 & 0.3725 & 0.3676 \\
\hline 1000 & 3504 & 3016 & 2936 & 2760 & 2760 & 0.2854 & 0.3316 & 0.3406 & 0.3623 & 0.3623 \\
\hline 1100 & 3516 & 3104 & 3242 & 2974 & 2978 & 0.3129 & 0.3544 & 0.3393 & 0.3699 & 0.3694 \\
\hline 1200 & 3800 & 3288 & 3494 & 3230 & 3230 & 0.3158 & 0.3650 & 0.3434 & 0.3715 & 0.3715 \\
\hline 1300 & 4308 & 3552 & 3814 & 3552 & 3550 & 0.3018 & 0.3660 & 0.3408 & 0.3660 & 0.3662 \\
\hline 1400 & 4688 & 3760 & 4112 & 3760 & 3760 & 0.2986 & 0.3723 & 0.3405 & 0.3723 & 0.3723 \\
\hline
\end{tabular}

Table 12 Percentage improvement of the proposed PCT128, PCT256, and PCT-E versus existing EDFSA (ED) and DFSA (D) techniques

\begin{tabular}{|c|c|c|c|c|c|c|}
\hline & \multicolumn{2}{|c|}{ PCT128 } & \multicolumn{2}{c|}{ PCT256 } & \multicolumn{2}{c|}{ PCT-E } \\
\hline & $\begin{array}{c}\text { improved } \\
\text { from ED }\end{array}$ & $\begin{array}{c}\text { improved } \\
\text { from D }\end{array}$ & $\begin{array}{c}\text { improved } \\
\text { from ED }\end{array}$ & $\begin{array}{c}\text { improved } \\
\text { from D }\end{array}$ & $\begin{array}{c}\text { improved } \\
\text { from ED }\end{array}$ & $\begin{array}{c}\text { improved } \\
\text { from D }\end{array}$ \\
\hline $\mathbf{1 0 0}$ & 16.48 & 16.48 & 0.00 & 0.00 & 0.00 & 0.00 \\
$\mathbf{2 0 0}$ & -9.70 & -9.70 & 0.00 & 0.00 & 0.00 & 0.00 \\
$\mathbf{3 0 0}$ & 0.00 & 0.00 & 0.00 & 0.00 & 0.00 & 0.00 \\
$\mathbf{4 0 0}$ & 3.10 & 1.17 & 10.62 & 8.83 & 10.29 & 8.50 \\
$\mathbf{5 0 0}$ & -2.13 & -5.12 & 4.12 & 1.32 & 4.12 & 1.32 \\
$\mathbf{6 0 0}$ & -5.14 & 1.01 & 0.00 & 5.86 & 0.12 & 5.97 \\
$\mathbf{7 0 0}$ & -9.36 & 7.55 & 0.00 & 15.47 & 0.00 & 15.47 \\
$\mathbf{8 0 0}$ & 7.33 & 9.80 & 14.41 & 16.69 & 14.41 & 16.69 \\
$\mathbf{9 0 0}$ & 1.05 & 5.97 & 9.58 & 14.08 & 8.38 & 12.94 \\
$\mathbf{1 0 0 0}$ & 2.65 & 16.21 & 8.49 & 21.23 & 8.49 & 21.23 \\
$\mathbf{1 1 0 0}$ & -4.45 & 7.79 & 4.19 & 15.42 & 4.06 & 15.30 \\
$\mathbf{1 2 0 0}$ & -6.27 & 8.05 & 1.76 & 15.00 & 1.76 & 15.00 \\
$\mathbf{1 3 0 0}$ & -7.38 & 11.47 & 0.00 & 17.55 & 0.06 & 17.60 \\
$\mathbf{1 4 0 0}$ & -9.36 & 12.29 & 0.00 & 19.80 & 0.00 & 19.80 \\
\hline Average & -1.66 & 5.93 & 3.80 & 10.80 & 3.69 & 10.70 \\
\hline
\end{tabular}

a better performance than the EDFSA by about 4 percent on average, while it is approximately 11 percent better than the DFSA approach as demonstrated in Figure 5. The optimal percentage of improvement of PCT256 method can achieve up to 14 percent and 21 percent compared with the EDFSA and DFSA respectively, depending on the number of tags within the interrogation zone. Nevertheless, the PCT-E method required additional number of groups from the PCT method and acquired slightly lower percentage of improvement compared with the PCT method. On the other hand, the PCT128 has a better performance than the DFSA method by around 6 percent on average, but does not show any improvement from the EDFSA technique, as displayed in Table 12. However, the PCT128 still shows some improvement in some cases and able to achieve up to 16 percent compared with the EDFSA and DFSA methods. Therefore, we conclude that our proposed PCT256 method is the most effective method in terms of system efficiency and number of slots minimisation.

\section{Conclusion}

Throughout this research, we have identified the significance of RFID tag anti-collision and developed a concept to minimise the tag starvation problem. We have proposed the Probabilistic Cluster-Base Technique (PCT) to maximise the performance efficiency and reduce the total number of slots and frames queried during the tag identification process.

From the results and analysis, we have found that both our proposed PCT256 and PCT-E methods have produced a minimal number of slots when compared with existing state-of-the-art approaches. Specifically, the PCT256 displays higher performance efficiency than the EDFSA by about 4 percent on average, while it is 


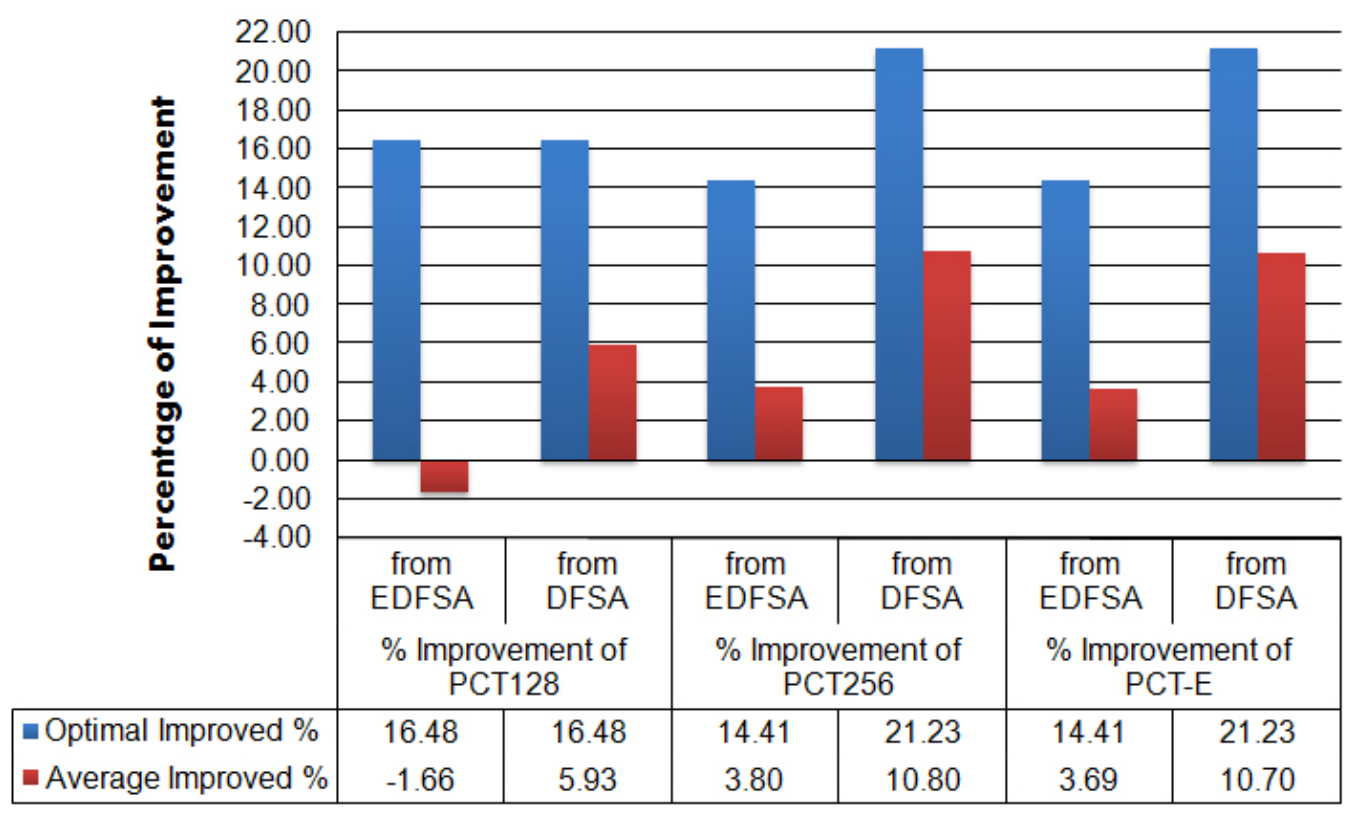

Fig. 5 Percentage of improvement of PCT compared to DFSA and EDFSA methods

approximately 11 percent better than the DFSA approach. The PCT-E method required an additional number of groups and acquired a slightly lower percentage of improvement compared with the PCT256 configuration. Through our empirical evaluation, we have concluded that our PCT256 method is the most reliable method. It has maintained its system efficiency above other existing state-of-the-art approaches and has the highest achieving performance.

\section{References}

1. Abramson, N.: The ALOHA System - Another Alternative for Computer Communications. In: Proceedings of Fall Joint Computer Conference, AFIPS Conference, pp. 281-285. Houston, Texas (1970)

2. Chen, W.T.: An Efficient Anti-Collision Method for Tag Identification in a RFID System. IEICE Transactions 89-B(12), 3386-3392 (2006)

3. Cho, H., Lee, W., Baek, Y.: LDFSA: A Learning-Based Dynamic Framed Slotted ALOHA for Collision Arbitration in Active RFID Systems. In: Advances in Grid and Pervasive Computing Second International Conference, vol. 4459, pp. 655-665. Springer Berlin/Heidelberg, Paris, France (2007)

4. Darcy, P., Stantic, B., Sattar, A.: Correcting Missing Data Anomalies with Clausal Defeasible Logic. In: Advances in Databases and Information Systems (ADBIS), Lecture Notes in Computer Science, vol. 6295, pp. 149$163(2010)$

5. Devarapalli, M.R., Sarangan, V., Radhakrishnan, S.: AFSA: an efficient framework for fast RFID tag reading in dense environments. In: QSHINE '07: The Fourth International Conference on Heterogeneous Networking for Quality, Reliability, Security and Robustness Workshops, pp. 1-7. ACM, New York, NY, USA (2007). DOI http://doi.acm.org/10.1145/1577222.1577242
6. Ding, J., Liu, F.: Novel Tag Anti-Collision Algorithm with Adaptive Grouping. Wireless Sensor Network (WSN) 1(5), 475-481 (2009). DOI 10.4236/wsn.2009.15057

7. Fan, X., Song, I., Chang, K.: Gen2-based hybrid tag anticollision $\mathrm{Q}$ algorithm using Chebyshev's inequality for passive RFID systems. In: Personal, Indoor and Mobile Radio Communications, 2008. PIMRC 2008. IEEE 19th International Symposium on, pp. 1-5. Cannes, France (2008). DOI 10.1109/PIMRC.2008.4699508

8. Fan, X., Song, I., Chang, K., Shin, D.B., Lee, H.S., Pyo, C.S., Chae, J.S.: Gen2-Based Tag Anti-collision Algorithms Using Chebyshev's Inequality and Adjustable Frame Size. pp. 653-662 (2008)

9. Floerkemeier, C.: Bayesian Transmission Strategy for Framed ALOHA Based RFID Protocols. In: RFID, 2007. IEEE International Conference on RFID Gaylord Texan Resort, pp. 228-235. Grapevine, TX, USA (2007)

10. Jain, S., Das, S.R.: Collision avoidance in a dense RFID network. In: WiNTECH '06: Proceedings of the 1st international workshop on Wireless network testbeds, experimental evaluation \& characterization, pp. 49-56. ACM, New York, NY, USA (2006)

11. Lee, C.W., Cho, H., Kim, S.W.: An Adaptive RFID AntiCollision Algorithm Based on Dynamic Framed ALOHA. IEICE Transactions 91-B(2), 641-645 (2008)

12. Lee, J.G., Hwang, S.J., Kim, S.W.: Performance Study of Anti-collision Algorithms for EPC-C1 Gen2 RFID Protocol. In: Information Networking. Towards Ubiquitous Networking and Services, vol. 5200/2008, pp. 523-532. Springer Berlin/Heidelberg, Estoril, Portugal (2008). DOI 10.1007/978-3-540-89524-4

13. Lee, S.R., Joo, S.D., Lee, C.W.: An enhanced dynamic framed slotted aloha algorithm for rfid tag identification. In: MOBIQUITOUS '05: Proceedings of The Second Annual International Conference on Mobile and Ubiquitous Systems: Networking and Services, pp. 166-174. IEEE Computer Society, Washington, DC, USA (2005) 
14. Lee, S.R., Lee, C.W.: An Enhanced Dynamic Framed Slotted ALOHA Anti-collision Algorithm . In: Emerging Directions in Embedded and Ubiquitous Computing, pp. 403-412. Springer Berlin/Heidelberg, Seoul, Korea (2006)

15. Li, B., Yang, Y., Wang, J.: Anti-collision Issue Analysis in Gen2 Protocol - Anti-collision issue analysis considering capture effect. Auto-ID Labs White Paper. (2009). Http://www.autoidlabs.org/singleview/dir/article/6/320/page.html

16. Myung, J., Lee, W.: Adaptive splitting protocols for RFID tag collision arbitration. In: MobiHoc '06: Proceedings of the 7th ACM international symposium on Mobile ad hoc networking and computing, pp. 202-213. ACM, New York, NY, USA (2006)

17. Pupunwiwat, P., Stantic, B.: Unified Q-ary Tree for RFID Tag Anti-Collision Resolution. In: A. Bouguettaya, $\mathrm{X}$. Lin (eds.) Twentieth Australasian Database Conference (ADC), CRPIT, vol. 92, pp. 47-56. ACS, Wellington, New Zealand (2009)

18. Pupunwiwat, P., Stantic, B.: Dynamic Framed-Slot ALOHA Anti-Collision using Precise Tag Estimation Scheme. In: H.T. Shen, A. Bouguettaya (eds.) TwentyFirst Australasian Database Conference (ADC), CRPIT, vol. 104, pp. 19-28. ACS, Brisbane, Australia (2010)

19. Pupunwiwat, P., Stantic, B.: Joined Q-ary Tree AntiCollision for Massive Tag Movement Distribution. In: B. Mans, M. Reynolds (eds.) Thirty-Third Australasian Computer Science Conference (ACSC), CRPIT, vol. 102, pp. 99-108. ACS, Brisbane, Australia (2010)

20. Quan, C.H., Hong, W.K., Kim, H.C.: Performance Analysis of Tag Anti-collision Algorithms for RFID Systems. In: Emerging Directions in Embedded and Ubiquitous Computing, vol. 4097, pp. 382-391. Springer Berlin/Heidelberg, Seoul, Korea (2006)

21. Ryu, J., Lee, H., Seok, Y., Kwon, T., Choi, Y.: A Hybrid Query Tree Protocol for Tag Collision Arbitration in RFID systems. In: MobiHoc '06: Proceedings of the 7th ACM international symposium on Mobile ad hoc networking and computing, pp. 5981-5986. IEEE Computer Society, Glasgow, UK (2007)

22. Schoute, F.C.: Dynamic Frame Length ALOHA. IEEE Transactions on Communications 31(4), 565-568 (1983)

23. Shin, J.D., Yeo, S.S., Kim, T.H., Kim, S.K.: Hybrid Tag Anti-collision Algorithms in RFID Systems. In: Computational Science ICCS 2007, vol. 4490, pp. 693-700. Springer Berlin/Heidelberg, Beijing, China (2007)

24. Vogt, H.: Efficient Object Identification with Passive RFID Tags. In: Pervasive '02: Proceedings of the First International Conference on Pervasive Computing, pp. 98-113. Springer-Verlag, London, UK (2002)

25. Wang, Y.Q., Jiang, G.P., Wang, J.: Framed slotted ALOHA with grouping tactic and binary selection for anti-collision in RFID systems. The Journal of China
Universities of Posts and Telecommunications 16(4), 4752 (2009). DOI 10.1016/S1005-8885(08)60247-2

26. Wang, Z., Liu, D., Zhou, X., Tan, X., Wang, J., Min, H.: Anti-collision Scheme Analysis of RFID System. Auto-ID Labs White Paper. (2007). Http://www.autoidlabs.org/singleview/dir/article/6/281/page.html

27. Zhu, L., Yum, P.T.: The optimization of framed aloha based RFID algorithms. In: MSWiM '09: Proceedings of the 12th ACM international conference on Modeling, analysis and simulation of wireless and mobile systems, pp. 221-228. ACM, New York, NY, USA (2009). DOI http://doi.acm.org/10.1145/1641804.1641842

\section{Author Biographies}

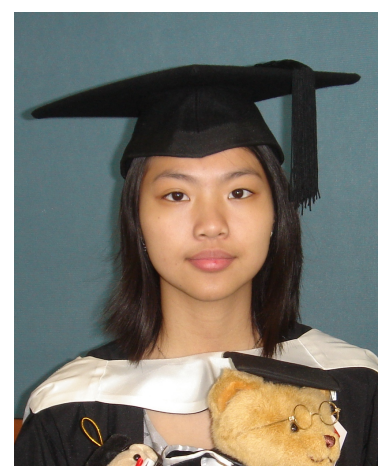

Prapassara Pupunwiwat received her Bachelor of Information Technology, in 2006, a Bachelor of Information Technology with First Class Honours, in 2007, and a Ph.D. in Information and Communication Technology, in 2012, from Griffith University, Australia. Her research interests include various aspects of Radio-Frequency Identification (RFID) technology, such as Anti-Collision algorithms, data streams errors management, and RFID business model strategies.

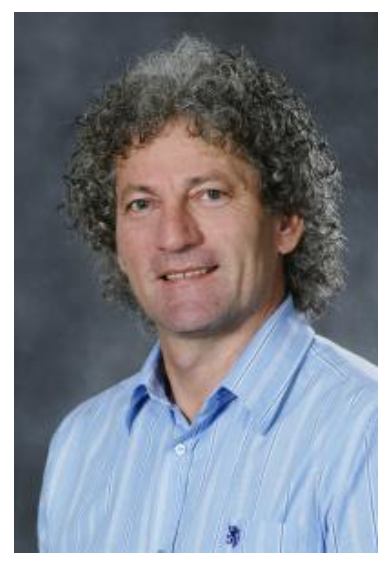

Bela Stantic is a Senior Lecturer at the School of Information and Communication Technology, Griffith University, and a member of the Institute for Integrated and Intelligent Systems (IIIS). Dr Stantic has been an academic staff member at Griffith University since 2001. His research interests include RFID systems, temporal and spatio-temporal databases,

bioinformatics, and database systems. 